\title{
A MOTOR INTERVENTION TO PREPARE LEARNING TO WRITE
}

\author{
Natalie Lavoie $^{1}$, \& Émile Lebel ${ }^{2}$ \\ ${ }^{1}$ Department of Education, University of Quebec in Rimouski (Canada) \\ ${ }^{2}$ Kinesiologist, Rimouski (Canada)
}

\begin{abstract}
Writing is a complex activity that requires the automation of graphomotor skills. Unfortunately, 10 to $30 \%$ of primary school students have difficulty at this level, which impairs the development of writing skills. It therefore seems judicious to intervene in kindergarten to support motor precision as well as visuomotor capacities, considered as prerequisites for writing by many researchers. The purpose of this study was to investigate the effect of motor training on visuomotor integration, motor precision and handwriting performance (speed and readability) in 5-year-old children. According to a quasi-experimental design (pretest, post-test with control group), 34 children participated in an intervention in subgroups, twice a week for 6 weeks. The mean and standard deviation were calculated for each of the tasks performed. Statistical tests ( $t$ test) were then carried out. The results show that the children in the experimental group improved their motor precision as well as their graphomotor skills compared to those in the control group. This project provides new insights into the benefits of working on basic skills in preparation for learning to write and will equip teachers on how to guide and support graphomotor skills before entering first grade.
\end{abstract}

Keywords: Motor intervention, handwriting, kindergarten.

\section{Introduction}

Writing is a complex activity that requires the automation of graphomotor skills in order to trace organized sets of letters. Difficulties at this level would impair the development of writing skills (Karlsdottir \& Stefansson, 2002). It is therefore important to find new ways to facilitate this learning from kindergarten since the ability to control the motor gesture has an impact on the fluidity of writing (readability and speed) (Danna \& Veley, 2015).

Handwriting is described as a perceptual (related to the shape of the letter) and motor skill (related to the movement producing the letter trajectory) which requires motor precision (control and coordination of the musculature of the hands and fingers) as well as visuomotor abilities (coordination between visual perception and finger movements), considered as prerequisites for writing by many researchers (Feder et Majnemer, 2007). Problems with any of these underlying components could lead to inefficient letter formation (Schneck et al., 2012) and on the ability to produce one's first name (Puranik \& Lonigan, 2012). Several studies have underlined the effectiveness of intervention programs that target these components among young students (Bazyk et al., 2009) but most of them were conducted in first grade or among children with disabilities. So, the purpose of this study was to investigate the effect of motor training on visuomotor integration, motor coordination and handwriting performance (speed) of general education kindergarten students (5-year-old children).

\section{Method}

\subsection{Participants}

According to a quasi-experimental design (pretest, post-test with control group), four kindergarten classrooms participated in the study: school 1: 2 intervention classrooms $(n=34)$; school 2 : 2 control classrooms $(\mathrm{n}=25)$. All kindergarten were invited to participate in this study. Signed parents ssente were obtained for all study participants. Consent was also obtained from school principals and teachers. For ethical reasons, the teachers of the control classrooms received the training program after the study period was complete. 


\subsection{Measures}

Visual-motor skills (VMI) and motor coordination (MC) were assessed with two tasks of the Beery-VMI, short form (Berry, Berry \& Buktenica, 2010). This test captures how visual perception and finger-hand movements are coordinated during handwriting. For the first task, the VMI, participants had to copy geometric forms. They get a point for each form completed correctly (max. score: 21). In the second task, the MC, participants traced a geometric form by connecting the dots without crossing the double-lined path. They had five minutes to complete as many as possible (max. score: 30 ). The raw score for the two tests were converted by age into a normalized score. Handwriting performance has been verified by a first name (FN) writing task (speed). Children had to write their first name as fast as possible, ensuring every letter was readable. The number of letters produced per second was calculated.

\subsection{Intervention}

Experimental group- In order to improve the motor skills required when writing, a training program was deployed. The intervention was supervised by a trained student in Kinesiology who went to the experimental classes. The program consists in 12 sessions (twice a week) each lasting for $30 \mathrm{~min}$. (total intervention time $=12$ hours). Each training session follows a well-defined plan, made up of 4 steps: 1- 5-min warm-up; 2- 10-min muscle building; 3- 10-min coordination activities; 4- 5-min cool-down.

The intervention was provided in sub groups (on average 8 students per group) in quiet, private rooms at the schools.

Control group- The children in this group received no intervention and attended their normal classroom activities. They participated in both pretest and posttest evaluation sessions at the same time as the children in the intervention groups.

\subsection{Data collection}

Data were collected a week before (pretest: February) and after training (posttest: April) using the same assessment battery. All data were collected by two students who were trained in administering the assessments. The children were met individually, in a room outside the classroom. The average duration of the meeting was around 15 minutes.

\subsection{Data analysis}

Two paired sample student t-tests were used to analyze mean differences in the pretest, posttest, and gain (posttest-pretest) scores for the control and experimental groups. A significance level of 5\% was used to determine statistically significant results.

\section{Results}

Before the start of the intervention, the scores of the experimental group and those of the control group were comparable on the three tests (VMI. MC, FN) ( $p>.05)$. After the intervention (T2), the results all improved for the experimental group while for the control group they increased only on the FN (decrease on the VMI and on the MC). Despite this progression of the experimental group, the differences between the groups are not significant.

However, we can observe, for the VMI, that the initial score of the experimental group (99.80) is slightly below the average of the standardized scores established for this test, i.e. 100, while that of the control group (101.44) is above (Table 1). At T2, we observe that the scores have tilted. That of the experimental group went above (102.62) while that of the control group slipped below (99.80).

Table 1. Mean (SD) Pretest and Posttest.

\begin{tabular}{lllll} 
& \multicolumn{2}{l}{ Intervention group $(n=34)$} & & \multicolumn{2}{l}{ Control group $(n=25)$} \\
& T1 & $\mathrm{T} 2$ & $\mathrm{~T} 1$ & $\mathrm{~T} 2$ \\
& $M(S D)$ & $M(S D)$ & $M(S D)$ & $M(S D)$ \\
\hline VMI (visuo-motor) & $99,80(11,31)$ & $102,62(6,22)$ & $101,44(6,19)$ & $99,80(11,31)$ \\
MC (motor coor.) & $103,63(10,00)$ & $105,53(10,26)$ & $101,52(9,35)$ & $100,64(11,28)$ \\
FN (handwriting) & $0,33(0,15)$ & $0,38(0,16)$ & $0,32(0,17)$ & $0,36(0,18)$ \\
\hline
\end{tabular}

Secondary Analysis

Another way to observe the results is by determining individual progress made by children. The difference between the pretest and the posttest was calculated for each child to observe changes in their level of performance. It is observed that $35,3 \%$ of the children in the experimental group improved on the three tests while only $12 \%$ of those in the control group progressed. 


\section{Discussion}

The finding that experimental group improved from pretest to posttest suggests a positive practice effect may have occurred, although the results are not significant, after a relatively short period of intervention. These results did not support the findings of some studies who found significant improvements after an abbreviated intervention period (Ohl et al., 2013) but are consistent with the findings of others who have found insignificant improvement in scores (Ducharme-Lapointe, 2005). Thus, the duration of the intervention should be reconsidered in order to obtain significant results. The small sample size may also account for the failure of the results to reach statistical significance. For the VMI, the experimental group went from a score below the normalized mean established to a score above, showing an evolution at a rate exceeding that of maturation. This result therefore suggests that improvement is not only linked to maturation but also to intervention. Various studies indicate that the score obtained on the Berry VMI is an important indicator of writing skills (Ziviani \& Wallen, 2005), hence the relevance of offering children a motor program before entering first year.

On the first name writing task, both groups show a stable production speed. Frequent writing practices in the natural context of the classroom contribute to the development of better motor control (Danna \& Veley, 2005) and are necessary for benefits to be observed when writing the first name. The program alone therefore could not influence the production speed. In addition, the relatively short time between the pretest and the posttest could also explain that the results of the two groups remained stable. A longer period seems essential for the integration of letters in memory and for a faster production of them (Vander Hart et al., 2010). It is interesting to note that already more than a third of the children who have benefited from the program would have the necessary skills to begin learning to write letters, hence the relevance of the experienced program.

\section{Conclusion}

Effectiveness of the intervention was not demonstrated in this study. However, the results are encouraging since already after 6 weeks progress can be observed. This program could be integrated into the activities of the kindergarten class to enhance the content relating to the development of graphomotor skills. Thus, children could start the first year better prepared. Eventually, it would be relevant to experiment the program over a longer period, with a larger sample.

\section{References}

Bazyk, S., Michaud, P., Goodman, G., Papp, P., Hawkins, E. \& Welch, M. A. (2009). Integrating occupational therapy services in a kindergarten curriculum: A look at the outcomes. American Journal of Occupational Therapy, 63, 160-171.

Beery, K. E., Beery, N. A. \& Buktenica, N. A. (2010). The Beery-Buktenica Developmental Test of Visual-Motor Integration ( $6^{\text {th }}$ ed.). Minneapolis: NCS Pearson.

Danna, J. \& Velay, J.L. (2015). Basic and supplementary sensory feedback in handwriting. Frontiers in Psychology, 6(169). doi: 10.3389/fpsyg.2015.00169

Ducharme-Lapointe, A. (2005). Les habiletés préparatoires à l'écriture: programme d'intervention novateur en ergothérapie. Mémoire de maitrise, Faculté de médecine, Université Laval, Québec.

Feder, K.P. \& Majnemer, A. (2007). Handwriting development, competency and intervention. Developmental medecine and child neurology, 49(4), 312-317.

Karlsdottir, R. \& Stefansson, T. (2002). Problems in developing functional handwriting. Perceptual and motor skills, 94(2), 623-662.

Ohl, A.M., Graze, H., Weber, K., Kenny,S., Salvatore, C. \& Wagreich, S. (2013). Effectiveness of a 10-Week Tier-1 Response to Intervention Program in Improving Fine Motor and Visual-Motor Skills in General Education Kindergarten Students, American Journal of Occupational Therapy, 67(5), 507-514.

Puranik, C.S. \& Lonigan, C.J. (2012). Name-writing proficiency, not length of name, is associated with preschool children's emergent literacy skills. Early Childhood Research Quarterly, 27(2): 284-294.

Schneck, C., Shasby, S., Myers, C. \& DePoy Smith, M.L. (2012). Handwriting Without Tears versus Teacher-Designed Handwriting Instruction in First Grade Classrooms. Journal of Occupational Therapy, Schools \& Early Intervention, 5(1), 31-42

Vander Hart, N., Fitzpatrick, P. \& Cortesa, C. (2010). In-depth analysis of handwriting curriculum and instruction in four kindergarten classrooms. Reading and Writing, 23, 673-699.

Ziviani, J. \& Wallen, M. (2006). The development of graphomotor skills. In A. Henderson \& C. Pehoski (Ed.), Hand function in the child: foundation for remediation (p.217-238). St-Louis, MI: Mosby Elsevier. 